\title{
A INOVAÇÃO NA PERSPECTIVA DE DIRETORES DE HOTÉIS EM AVEIRO - PORTUGAL
}

\author{
INNOVATION IN THE PERSPECTIVE OF HOTEL DIRECTORS OF AVEIRO - PORTUGAL
}

\section{LA INNOVACIÓN EN LA PERSPECTIVA DE DIRECTORES DE HOTELES EN AVEIRO - PORTUGAL}

\author{
Jussara Danielle Martins Aires \\ Mestre em Ciências Sociais - Universidade Federal do Rio Grande do Norte (UFRN) \\ Doutoranda em Turismo - Universidade de Aveiro \\ jussaradma@ua.pt
}

Data de Submissão: 22/01/2017

Data de Aceitação: 03/08/2017

RESUMO: Os estudos sobre inovação em hotéis merecem atenção especial por serem essas empresas consideradas sine qua non para o funcionamento da atividade turística. No entanto, ainda há escassez de pesquisas (sobretudo, qualitativas) contemplando tal temática em destinos, cujo fluxo turístico tem crescido, como é o caso de Aveiro. Assim, este artigo objetiva mostrar a concepção de inovação sob a ótica de diretores hoteleiros nesta cidade, evidenciando mais precisamente o conceito, a importância e a implementação de inovações nos últimos três anos na perspectiva destes profissionais. Utilizou-se um guião de entrevista com questões abertas. A amostra, escolhida por conveniência, foi composta por quatro diretores, indicados pela técnica chamada "bola de neve". Para apreciar os dados, empregou-se a análise de conteúdo. Percebeu-se que a criação e/ou melhoria de serviços é principalmente resultante de sugestões de colaboradores, parcerias, captação de necessidades obtidas junto a clientes, pressão de autoridades, participação em eventos e são também influenciadas por razões ambientais. As inovações citadas, representadas basicamente por pequenas mudanças (melhorias) e reparos infraestruturais, são consideradas pelos directores algo positivo e há uma preocupação em implementá-las constantemente. A pesquisa permitiu o levantamento de informações importantes a serem consideradas no processo de medição da inovação em hotéis.

PALAVRAS-CHAVE: Inovação; Percepção; Diretores hoteleiros em Aveiro.

\begin{abstract}
Studies on innovation in hotels deserve special attention because these companies are considered sine qua non for the operationalization of the tourism sector. However, there is still a lack of research (particularly qualitative) on this subject in destinations where tourism flow has increased, as is the case of Aveiro. This article therefore aims to show the concept of innovation from the perspective of hotel directors of this city, showing, more precisely, the concept, importance and implementation of innovations in the last three years. An interview script with open questions was used. The sample, chosen by convenience, was comprised of four directors, indicated by the "snowball" technique. For the data analysis, content analysis was used. It was observed that the creation and/or improvement of services are mainly the result of suggestions or ideas from employees, partnerships, information about requirements obtained from clients, pressure from authorities, and participation in events. They are also influenced by environmental factors. The innovations cited, which are basically represented by small changes (improvements) and infrastructure repairs, are considered by the directors as something positive, and there is a concern to implement them constantly. This research enabled the collection of important information, to be considered in the process of measuring innovation in hotels.
\end{abstract}

KEYWORDS: Innovation; Perception; Hotel management in Aveiro.

RESUMEN: Los estudios sobre innovación en hoteles merecen atención especial puesto que estas empresas son consideradas sine qua non para el funcionamiento de la actividad turística. Sin embargo, todavía son escasas las investigaciones (sobre todo cualitativas) que contemplan tal temática en destinos cuyo flujo turístico ha crecido, como es el caso de Aveiro. Así, el objetivo de este artículo es mostrar la concepción de innovación desde el punto de vista de los directores hoteleros en esta ciudad, evidenciando con más precisión el concepto, la importancia y la implementación de innovaciones en los últimos tres años en la perspectiva de estos profesionales. Se utilizó un guión de entrevista con 
preguntas abiertas. La muestra, elegida por conveniencia, estuvo compuesta por cuatro directores, indicados mediante la técnica denominada "bola de nieve". Para apreciar los datos, se empleó el análisis de contenido. Se observó que la creación y/o mejoría de servicios es principalmente resultado de sugerencias de colaboradores, alianzas, captación de las necesidades de los clientes, presión de las autoridades, participación en eventos, y que también reciben influencias de los factores ambientales. Las innovaciones citadas, representadas básicamente por pequeños cambios (mejorías) y reparos infraestructurales, son consideradas por los directores como algo positivo y hay una preocupación por implementarlas constantemente. La investigación permitió conocer informaciones importantes a ser consideradas en el proceso de medición de la innovación en hoteles.

PALABRAS CLAVE: Innovación; Percepción; Directores hoteleros en Aveiro.

INTRODUÇÃO

inovação, diretamente relacionada a habilidades, investimentos,
empresas e competitividade, constitui um dos principais
impulsionadores da produtividade na economia. Também no turismo, um dos principais sectores econômicos do mundo, a competitividade e o progresso das empresas, em grande medida, dependem das suas atividades de inovação.

Surpreendentemente, a pesquisa sobre inovação no turismo até agora recebeu pouca atenção dos estudiosos, de forma que a indisponibilidade de informações relevantes para consolidar esse campo de investigação é enfatizada na literatura (Jacob, Tintoré, Aguiló, Bravo \& Mulet, 2003; Pikkemaat \& Weiermair, 2007; Orfila-sintes \& Mattsson, 2009; Orfila-Sintes et al., 2005; Hjalager, 2010; Pivcevic \& Pranicevic, 2012; Krizaj, Brodnik \& Bukovec, 2014).

Ao analisar as poucas pesquisas existentes, percebe-se que uma maior ênfase tem sido dada ao ramo de hospedagem por este ser considerado essencial ou condição sine qua non à própria existência do turismo como setor (Orfila-sintes, Crespí-Cladera \& Martínez-Ros, 2015; Pikkemaat \& Peters, 2006; Orfila-sintes \& Mattsson, 2009; Pivcevic \& Pranicevic, 2012; Tejada \& Moreno, 2013; Thomas $\&$ Wood, 2014).

No entanto, aplicadas a contextos específicos, tais pesquisas utilizam na grande maioria dos casos métodos de coleta de dados de natureza quantitativa (principalmente questionários) face à tentativa de averiguar como e/ou em que medida ocorre a inovação no referido ramo. Destarte, a perspectiva dos directores hoteleiros, que desempenham papel fundamental no processo de implementação e difusão da inovação organizacional, é desconsiderada. 
Investigar os aspectos subjectivos envolvidos nos processos de inovação dessas empresas é mister e, para efeitos desse estudo, apoia-se no princípio de que a implementação e a continuidade da inovação hoteleira (independente do porte ou dimensão) são condicionadas à percepção e ao grau de importância que seus directores atribuem a tal fenômeno.

Em Portugal, o turismo cresce e acompanhando esse crescimento, a cidade de Aveiro, situada na Região Central do país, tem apresentado, nos últimos anos, um aumento crescente da demanda de turistas nacionais e internacionais. Na referida cidade, o número de dormidas registrou um crescimento superior a $40 \%$ entre 2015 e 2016 e as previsões futuras são favoráveis (Camara de Aveiro, 2017).

Com base nessas considerações, este trabalho tem como objetivo mostrar a concepção de inovação sob a ótica de diretores hoteleiros em Aveiro, evidenciando mais precisamente o conceito, a importância e a implementação de inovações nos últimos três anos na perspectiva destes profissionais. Em segundo plano, manifestou-se o interesse em revelar se clientes, colaboradores, parceiros e fontes externas do conhecimento exercem influências na implementação dessas inovações.

Os procedimentos metodológicos utilizados para alcançar tal propósito caracterizam-se como qualitativos e exploratórios. Entrevistas foram realizadas, seguindo um guião com questões abertas direcionadas à amostra (escolhida por conveniência) composta por quatro diretores, indicados pela técnica chamada "bola de neve", por meio da qual um entrevistado indica o próximo e assim por diante. Os dados, por sua vez, foram apreciados seguindo os princípios da Análise de Conteúdo, com vistas a compreender criticamente o sentido da comunicação e as significações explícitas ou ocultas (Chizzotti, 2006).

Este artigo está estruturado da seguinte forma: a seguir, será apresentada uma fundamentação teórica, que discute o conceito de inovação, sua aplicação e suas particularidades no sector de turismo e, mais especificamente, na hotelaria. Na terceira secção, os procedimentos metodológicos são apresentados, contemplando o design da pesquisa, os aspectos de população (e amostra), a coleta de dados (instrumento), o tratamento e a análise de dados. Na seção seguinte, os resultados são então discutidos e confrontados 
com a teoria apresentada ao mesmo tempo em que as hipóteses são testadas. Consecutivamente, as conclusões recapitulam e apontam as contribuições e as limitações do trabalho, além de fazer recomendações para pesquisas futuras.

\section{REVISÃO DA LITERATURA}

\section{INOVAÇÃO: CONCEITOS E APLICAÇÃO NO TURISMO}

Existem distintos conceitos de inovação, que variam em função da abordagem, área científica e domínio de aplicação. O economista austríaco, Schumpeter (1934), destacou a relevância da inovação, apontando-a como determinante imprescindível do desenvolvimento socioeconômico de um país. De acordo com a perspectiva Schumpeteriana, é atribuída à figura do empreendedor toda a responsabilidade e poder para a mudança e progresso econômico por meio da inovação. Esta, por sua vez, foi definida por ele como a criação elou desenvolvimento de algo novo. Fenômeno esse que pode ser aplicado a: um produto ou melhoria significativa num produto, um novo método de produção, abertura de mercado, uma nova fonte de fornecimento, adoção de uma nova forma de organização (Schumpeter, 1934).

Tal conceito claramente revela a inovação como um resultado ou desempenho e, nesse sentido, Hall \& Williams (2008) destacam dois aspectos através dos quais ela pode ser descrita: a variedade de suas formas (tipos) e de seus impactos (mundial, regional, sectorial ou até mesmo empresarial).

A Organização para a Cooperação e Desenvolvimento Econômico (OCDE), numa perspectiva muito semelhante à de Schumpeter, deu uma contribuiç̧ão fundamental, buscando uniformizar a aplicação do conceito aos diferentes setores econômicos, por meio do Manual de Oslo, de acordo com o qual a inovação "consiste na implementação de um produto (bem ou serviço) novo ou significativamente melhorado, ou um processo, um novo método de marketing, ou um novo método organizacional nas práticas de negócios, na organização do local de trabalho ou nas relações externas." (OCDE, 2005, p.46). 
De modo geral, o conceito de inovação no turismo apresentado na literatura baseia-se, pois, nessas abordagens, que segundo Gomezelj (2016), Camisón e Monfort-Mir (2012), aplica-se perfeitamente à indústria manufatureira e é proveniente dela. O turismo é um campo de investigação relativamente novo (Hjalager, 2010) e a pesquisa sobre inovação em serviços também o é. De facto, o turismo não constitui um sector no qual podem ser encontrados os mesmos padrões e classificações da indústria de produção de bens ou manufatureira (Camisón \& Monforth-Mir, 2012; Pivcevic \& Pranicevic, 2012).

O turismo é um setor de natureza fragmentada, com predominância de pequenas empresas (Hjalager, 2010). A maioria das empresas desse setor é ou está associada essencialmente a "serviços", conforme pondera Dwyer, Forsyth, \& Dwyer (2010). Essa característica traz à tona elementos como a intangibilidade, com destaque para a importância das relações pessoais e a experiência turística, cuja incerteza, os sinais prévios de qualidade de serviço e a viagem para os locais a visitar são determinantes.

Outras características econômicas do turismo são explicitadas por Silva, Matias, \& Pintassilgo (2014): a interdependência, para a qual se chama atenção para o fato de os produtos turísticos e o seu usufruto pelos consumidores não serem oferecidos isoladamente; a rigidez espacial, em que se destaca a importância da localização da oferta e a necessidade de o consumidor se deslocar, e não o produto; e, ainda, o caráter sazonal, no tempo e no espaço, destas atividades, com inerentes efeitos, por exemplo, sobre as políticas de preços das empresas (Silva et al., 2014).

Com efeito, Hjalager (2010) observou que o turismo tem característicaschaves para desenvolver novas definições e tipologias de inovação e, então, propôs cinco diferentes tipos de inovação no turismo:

(i) Inovações de produto ou serviço (mudanças que podem ser observadas diretamente pelos turistas e outros clientes, com "novo" significado ou nunca antes visto ou simplesmente novo para a empresa ou destino.

(ii) Inovações de processo (tipicamente iniciativas de back-stage destinadas a melhorar a eficiência e a produtividade. Investimentos tecnológicos são os principais fatores de tais inovações). 
(iii) Inovações gerenciais (novas formas de organização dos processos de negócios, capacitação do pessoal, compensação do trabalho exemplar com benefícios financeiros ou não financeiros e melhoria da satisfação no local de trabalho. Os métodos de retenção de empregados são extremamente valiosos no setor de turismo).

(iv) Inovações de marketing (incluindo novos conceitos de marketing, tais como programas de fidelização e co-produção de marcas).

(v) Inovações institucionais (novas formas de estrutura colaborativa/ organizacional, tais como clusters, redes e alianças ou parcerias).

Essencialmente, essas são as dimensões de inovação usadas ou rearranjadas em vários estudos relacionados ao turismo. No entanto, é difícil distinguir claramente entre as cinco categorias de inovação (produto, processo, gestão, marketing e inovações institucionais), uma vez que existe uma estreita interação entre elas (Gomezelj, 2016).

A inovação apresenta comportamentos diferenciados entre os sectores de produção de bens, bens e serviços e mesmo dentro do próprio sector de serviços, em que o turismo está inserido e tem se tornado cada vez mais evidente (Hjalager, 2010; Camisón \& Monforth- Mir, 2012; Pikkemaat \& Peters, 2006; Jacob et al., 2003; Čivre \& Omerzel, 2015). Mesmo dentro do sector de turismo existe uma variedade de empresas e ramos (hospedagem, restauração, transportes, entretenimento e lazer, agenciamento/organização de viagens, etc.) que se diferenciam entre si, em termos de processos, dinâmicas de funcionamento e por conseguinte na maneira como se comportam perante a inovação (Camisón \& Monfort-Mir, 2012; Krizaj, Brodnik \& Bukovec, 2014).

Em menor ou em maior proporção, estudos revelam que a inovação impacta positivamente o desempenho das empresas (inclusive de hotéis). Desempenho este que será tão ou mais favorável à medida em que os seus líderes, a partir da consciência do que a inovação é e dos benefícios que esta pode trazer, mobilize esforços e pessoas para criar soluções (oferta de produto ou serviço novo ou melhorado, por exemplo), agregando valor a seus clientes (Schumpeter, 1934; Orfila-Sintes et al, 2005; Ulwick, 2005; Orfila- 
sintes \& Mattsson, 2009; Čivre \& Omerzel, 2015; Martínez-Román, Tamayo, Gamero \& Romero, 2015 Saunila, 2016)

\section{INOVAÇÃO EM MEIOS DE HOSPEDAGEM}

Considerando-se o setor de hospedagem, há três características principais que o distingue de outros serviços. Primeiro, a existência de uma classificação categórica regular por estrelas, que vai de 1 a 5 estrelas (ou mais), de modo a determinar o tipo, o número e a qualidade de serviços oferecidos aos hóspedes. Segundo, é muito comum que haja empresas especializadas no serviço de gestão hoteleira e muitas combinações podem surgir. Há hotéis em que os proprietários são os próprios diretores, ou há os casos em que profissionais executivos gerenciam hotéis alheios sob um contrato de gestão ou franquia. Terceiro, hotéis podem operar em cadeias, com uma matriz, que a depender da empresa possui diferentes atribuições e níveis variados de integração vertical. A implementação de inovações pode sofrer influências em função dessas características (Orfila-Sintes et al., 2005).

Uma vez que a direção do hotel conhece seu público-alvo e define estratégias para alcançá-los, inovações podem ser implementadas de forma contínua a partir do contato direto hotel-cliente. Devido ao conhecimento sobre as necessidades e os desejos desses clientes, a inovação é muitas vezes iniciada a partir das interacções pessoais. Assim, conforme poderam Jacob et al. (2003), Pikkemaat \& Peters (2006), Orfila-Sintes et al. (2005); Orfila-sintes \& Mattsson (2009), Hjalager (2010) e Camisón \& Monfort-Mir (2012), os recursos humanos são essenciais no sector de hospedagem. A qualificação de colaboradores deve ser uma preocupação constante dos diretores, já que esses colaboradores devem e costumam estar envolvidos em todos os processos de criação e implementação de inovações que ocorrem na empresa (Orfila-Sintes et al., 2005, Orfila-sintes \& Mattsson, 2009; Pivcevic \& Pranicevic, 2012).

Corroborando e complementando tais ideias, Ottenbacher \& Gnoth (2005) apontaram nove fatores que directores de hotéis devem considerar para implementar inovações de serviços bem-sucedidas: seleção de mercado; gestão estratégica de recursos humanos; treinamento de funcionários; capacidade de identificar e responder necessidades de mercado; gestão do conhecimento 
(empowerment); avaliação baseada em comportamentos; sinergia de mercado; equipe de colaboradores motivados e compromissados; qualidade tangível.

Com efeito, esses factores contemplam as características particulares do sector de turismo e hotelaria mostrados na literatura, em que se sobressai a importância dos recursos humanos. Além disso, as dimensões críticas da inovação em hotéis costumam ser contempladas em quatro grupos de interesses: (1) relacionado aos serviços ou aos produtos, (2) ao mercado, (3) relacionado a processos e (4) à organização.

Com base nisso, são identificados quatro tipos de inovação hoteleiras, decorrentes das características dos serviços e das próprias particularidades desse ramo: 1) gerencial, 2) comunicações externas, 3) escopo de serviços e 4) e de back-office (Orfila-Sintes et al., 2005, Orfila-sintes \& Mattsson, 2009).

O tipo de inovação gerencial refere-se à qualidade dos processos de gestão, às aplicações TIC para gestão e às melhorias da estrutura organizacional. Portanto, a inovação de gestão considera a importância da qualidade do ambiente na competitividade do hotel (Alvarez; Burgos; Jiménez; Céspedes; Lorente, 2001). As aplicações de TIC são proeminentes para tornar os processos de gestão eficientes (Camisón, 2000).

As empresas de conhecimento não intensivo, como é o caso das que se encontram no setor de hospedagem, inovam também através da introdução de Pesquisa \& Desenvolvimento (P\&D) incorporada em tecnologia (ou seja, pela aquisição de novos equipamentos ou software), em vez de realizar atividades internas de P\&D. A incorporação dessas tecnologias pode ser traduzida em uma vantagem competitiva, seja pela eficiência produtiva (redução de custos), seja pela capacidade aprimorada de diferenciação (melhorar o serviço prestado, adaptando-o às demandas dos clientes) e de modo a atender recomendações do Manual de Oslo (Hjalager 2002; Orfilasintes \& Mattsson, 2009)

Mudanças na estrutura organizacional tornam a inovação organizacional relevante nos serviços hoteleiros (Van der Aa, \& Elfring, 2002). Na verdade, os problemas gerenciais a serem solucionados por meio da inovação organizacional são cruciais nas organizações de serviços (Wright, 2002; Choi, Poon \& Davis, 
2008). Harrington (2004), citado por Orfila-sintes \& Mattsson (2009), descobriu que os diretores que podem utilizar a informação em tempo real das operações e o ambiente competitivo para avaliar simultaneamente vários cursos de ação mostraram melhorar o desempenho da empresa.

A inovação em comunicações externas retrata o elevado conteúdo informativo e intangível dos produtos e dos processos de serviços na indústria hoteleira. Nesse sentido, as TIC também desempenham um papel fundamental, tanto na geração de oportunidades como nos novos "serviços", e na revolução das formas como a maioria dos serviços "tradicionais" são fornecidos e divulgados. O uso, bem como a a atualização das TIC (por exemplo, Internet), é uma inovação muito relevante que ajuda a aumentar a competitividade das empresas porque facilita o gerenciamento de relacionamentos com clientes, por meio de um melhor e mais fácil intercâmbio de informações. Além disso, as TIC podem melhorar a eficiência das relações entre a empresa e os intermediários, fornecedores e outros stakeholders (Camisón, 2000; Orfilasintes \& Mattsson, 2009). Hotéis que pertençam a uma rede ou atuam em parcerias estreitam relações comerciais, aumentam possibilidades de vendas, reduzem riscos, custos e assim tendem a aumentar a sua capacidade de inovação (Tejada \& Moreno, 2013; Martínez-Román et al., 2015; Pikkemaat \& Peters, 2006).

A inovação no escopo de serviços é relevante devido à importância da prestação de serviços para a competitividade dos hotéis, uma vez que leva a mudanças na produção de serviços e, muitas vezes, à incorporação de ativos tecnológicos que melhoram a produção do serviço e os aspectos tangíveis na forma como é prestado. Stamboulis e Skayannis (2003), conforme citado por Orfila-sintes e Mattsson (2009), mostram que a inovação no âmbito do serviço é a parte mais tangível da inovação e, de fato, o núcleo do valor do cliente.

Por sua vez, a inovação de back-office consiste na incorporação de ativos tecnológicos novos para a melhoria da produtividade e para conseguir uma prestação de serviços mais eficiente. Muitos novos produtos na indústria hoteleira não são simplesmente produtos; eles também têm implicações no serviço. Eles também podem implicar modificação de processos de back-office ou no atendimento a clientes. Assim, o processo e o sistema de concepção 
e teste de um novo serviço abrangem todo o processo de entrega, não apenas o elemento experimentado pelo cliente, e muitas vezes envolve a reengenharia do processo de back-office (Orfila-sintes \& Mattsson, 2009), reforçando a forte interligação entre os tipos de inovação enfatizado por Gomezelj (2016).

Outro aspecto a ser considerado diz respeito às mudanças na oferta dos serviços por razões ambientais. Ações como redução do fluxo de água e aquisição de equipamentos que consomem menos energia têm sido frequentemente adotadas em hotéis e traduzem como formas de racionalizar recursos naturais, essencialmente reduzindo custos, além de melhorar a imagem do hotel. A implementação de inovações como essas pode surgir em decorrência de pressões de clientes, autoridades ou até mesmo para a obtenção de certificações (Le, Hollenhorst, Harris, Mclaughlin \& Shook, 2006).

Tether (2005) destaca mais uma particularidade que se aplica ao setor hoteleiro: em razão dos serviços serem frequentemente únicos, é difícil de identificar as diferenças entre as variações de serviços e as inovações propriamente ditas. $O$ que pode ser introduzido como uma nova variante de serviço, desenhada para a satisfação de uma determinada necessidade dos clientes, pode também posteriormente ser entendido como o começo de uma modificação nas atividades do serviço que levam a tomar um rumo diferente ao idealizado inicialmente (Tether, 2005). Pikkemaat \& Peters (2006) corroboram essa afirmação e complementam que a maioria das atividades de inovação constitui apenas mudanças superficiais, que inclui pequenos reparos na infraestrutura do hotel.

A identificação de atividades de inovação dentro do sector de hospedagem surge a partir de fontes diretas, dos próprios esforços de oferecer serviços aos hóspedes. O hotel é o lugar no qual o serviço é fornecido e consumido, no qual os diretores têm o incentivo a partir das pessoas com quem interagem no exercício de sua função. É diferente da indústria manufatureira, que utiliza patentes e registo de atividades de P\&D como principais indicadores de inovação (Camisón \& Monforth-Mir, 2012; Hjalager 2002; Hjalager 2010; Orfila-sintes \& Mattsson, 2009). 


\section{ABORDAGEM QUALITATIVA}

Hjalager (2010) destaca a importância de desenvolver estudos de casos do tipo exploratório com a utilização de métodos qualitativos. A abordagem qualitativa torna-se pertinente neste trabalho, pois além de favorecer a aproximação do investigador ao objeto pesquisado, também "garante a riqueza de dados e permite ver um fenômeno na sua totalidade" (Vieira, 2004, p.15). Estabeleceu-se que a pesquisa é exploratória, já que uma das suas primeiras etapas teve o intuito de desenvolver conceitos e ideias, proporcionando uma visão mais geral de um fato (Dencker, 1998). Na investigação qualitativa, a literatura deve ser usada de uma forma consistente com os pressupostos metodológicos (Marujo, 2013).

Miles e Huberman (1994) destacam que, nessa abordagem, pode haver maior riqueza de detalhes e aproximação com o meio investigado. O papel do pesquisador é capturar os dados por meio das percepções dos atores locais, suspendendo seus preconceitos sobre os tópicos pesquisados. Ainda segundo os autores, muitas interpretações do material são possíveis, relativamente pouca padronização instrumental é utilizada e a maioria das análises é feita utilizando palavras.

Para Creswell (1998), a pesquisa qualitativa é um processo de investigação por meio da qual é possível construir um quadro complexo e holístico, analisar palavras e reportar detalhadamente as visões de informantes.

Outra maneira de conceituar a pesquisa qualitativa pode dar-se por intermédio da definição dos tipos de dados ou informação que são classificados como qualitativos. Patton (2002) considera nesse rol de dados as entrevistas com citações verbalizadas para serem interpretadas, as observações e as descrições de campo detalhadas que incluem o contexto dessas observações e os documentos ou informaç̃es selecionadas de documentos que gravem e preservem o contexto.

Ao mencionar a entrevista como técnica privilegiada de comunicação e coleta de dados, Minayo (2010) enfatiza que se trata da estratégia mais 
utilizada no trabalho de campo e pode ser conceituada como "uma conversa a dois, ou entre vários interlocutores, realizada por iniciativa do entrevistador, destinada a construir informações pertinentes para um objeto de pesquisa, e abordagem pelo entrevistador, de temas igualmente pertinentes tendo em vista este objetivo" (Minayo, 2010, p.261).

\section{RECOLHA DE DADOS}

O estudo da percepção da inovação empresarial focou na indústria turística, aqui representada apenas por hotéis, por serem considerados os principais empreendimentos que a compõem. O setor de restauração é mais representativo (em quantidade) do que o de hospedagem, porém não há informações suficientes acerca do percentual de atendimento destes estabelecimentos a turistas, ao passo que nos hotéis o público-alvo é formado integralmente por não residentes.

A partir da elaboração prévia de um guião de entrevista, foram realizados encontrospresenciaisnosempreendimentosinvestigados(previamenteagendados) com cada um dos entrevistados, a saber, diretores de hotéis em Aveiro.

A escolha das questões abertas justifica-se pelo fato de existir um campo de conhecimento polissêmico sobre a inovação empresarial, que reflete na sua importância e na sua implementação por parte dos diretores das empresas e ainda por haver poucas evidências empíricas acerca da sua aplicação no turismo. Pretende-se, a partir das categorias que emergiram das respostas espontâneas, em contraponto com as categorias encontradas na literatura, ter o ponto de partida para elaborar as questões quantitativas para pesquisas futuras e realizar inquéritos junto à amostra representativa da população.

De acordo com o Instituto Nacional de Estatística - INE (2010), em Aveiro existem 16 estabelecimentos hoteleiros. Com base nessa informação, a amostra, escolhida por conveniência, foi composta por quatro diretores, representantes de hotéis de duas, três e quatro estrelas na referida cidade. Os entrevistados foram indicados pela técnica chamada "bola de neve", em que um entrevistado indica o próximo e assim por diante. O primeiro entrevistado foi indicado por uma docente do Programa Doutoral em Turismo da Universidade de Aveiro. 
As entrevistas foram realizadas nos dias 2, 4 e 7 de maio de 2016. Todos os entrevistados foram contatados pessoalmente para fazer o agendamento da entrevista. Todas as entrevistas foram gravadas (com o consentimento do entrevistado) e anotações também foram feitas.

As entrevistas foram transcritas, separadamente, cada qual devidamente nomeada em documento de formato Word. Em seguida, foram enviadas através de upload para fontes internas do WebQDA. O guião de entrevista, contendo 25 questões abertas, agrupadas em função dos objetivos, foi elaborado para a obtenção de respostas espontâneas, posteriormente analisadas em conformidade com a revisão da literatura. O número de questões a serem analisadas foi reduzido, dada a incapacidade de suporte do WebQDA e inconsistência de respostas. No momento das entrevistas, em algumas ocasiões, julgou-se necessário, explicar melhor as questões.

As questões 1 e 2 objetivaram mostrar a percepção dos diretores de hotéis quanto ao conceito e à importância da inovação, ao passo que as questões 3, 4 e 5 objetivaram identificar as inovações de produto ou de serviço implementadas no hotel. As questões de 6 a 10 buscaram saber se clientes, colaboradores, parceiros e outras fontes externas de conhecimento exercem influência para a implementação de inovações no hotel. Há, ainda, o intuito de identificar possíveis categorias de análise da inovação que não estejam contempladas na revisão de literatura.

Tabela 1: Sistematização das questões e objetivos.

\begin{tabular}{|c|c|c|}
\hline Objetivo & \multicolumn{2}{|c|}{ Mostrar a percepção dos diretores de hotéis quanto ao conceito e à importância da inovação } \\
\hline \multicolumn{2}{|r|}{ Questões } & Autores \\
\hline \multicolumn{2}{|c|}{$\begin{array}{l}\text { O que o Sr.(a) entende por inovação? } \\
\text { Qual a importância que tem a inovação para o hotel? }\end{array}$} & $\begin{array}{l}\text { Schumpeter (1934); OCDE (2005); Hjalager } \\
\text { (2010); Hjalager (2002) Camisón \& } \\
\text { Monfort-Mir (2012); Hall e Williams (2008); } \\
\text { Le et al. (2006); Čivre \& Omerzel (2015); } \\
\text { Martínez-Román et al. (2015); Saunila } \\
\text { (2016); Orfila-Sintes et al. (2005); Ulwick, } \\
\text { (2005); Orfila-sintes \& Mattsson (2009). }\end{array}$ \\
\hline Objetivo & \multicolumn{2}{|c|}{ Identificar as inovações de produto ou serviço implementadas no hotel } \\
\hline \multicolumn{2}{|r|}{ Questões } & Autores \\
\hline $\begin{array}{l}\text { A empresa } \\
\text { Melhorou de }\end{array}$ & $\begin{array}{l}\text { Nos últimos três anos: } \\
\text { çou com sucesso, algum novo produto ou } \\
\text { serviço? Se sim, quais? } \\
\text { ma significativa algum serviço existente? Se } \\
\text { sim, de que forma? }\end{array}$ & $\begin{array}{l}\text { Jacob et al (2003); OCDE (2005); Orfila-sintes } \\
\text { et al. (2005); Pikkemaat \& Peters (2006); Le } \\
\text { et al (2006); Orfila-sintes \& Mattsson (2009); } \\
\text { Hjalager (2010); Hjalager (2002); Camisón } \\
\text { \& Monfort-Mir (2012); Camisón (2000); } \\
\text { Pivcevic \& Pranicevic, 2012). }\end{array}$ \\
\hline
\end{tabular}


Dor: 10.14210/rtva.v19n3.p487-512

\begin{tabular}{|c|c|c|}
\hline Objetivo & \multicolumn{2}{|c|}{$\begin{array}{l}\text { Perceber a influência que clientes, colaboradores, parceiros e fontes externas do } \\
\text { conhecimento exercem para a implementação de inovações no hotel }\end{array}$} \\
\hline & Questões & \\
\hline $\begin{array}{r}\text { Algum servi } \\
\text { das necessic } \\
\text { Alguma in } \\
\text { deco } \\
\text { Fez novas pa } \\
\text { A empresa fa } \\
\text { eventos } \\
\text { imple }\end{array}$ & $\begin{array}{l}\text { Jos últimos três anos: } \\
\text { criado ou melhorado em consequência } \\
\text { dentificadas junto aos clientes? Quais? } \\
\text { radores recebem formação? } \\
\text { o foi implementada com sucesso em } \\
\text { da sugestão de colaboradores? } \\
\text { que originaram a criação ou a melhoria } \\
\text { novos serviços? Quais? } \\
\text { de consultoria ou recorre a instituições, } \\
\text { iações para obter conhecimento e } \\
\text { r inovações na empresa? Quais? }\end{array}$ & $\begin{array}{l}\text { OCDE (2005); Orfila-Sintes et al. (2005); } \\
\text { Pikkemaat \& Peters (2006); Orfila-sintes \& } \\
\text { Mattsson (2009); Hjalager (2010); Hjalager } \\
\text { (2002); Camisón \& Monfort-Mir(2012); } \\
\text { Camison (2000); Čivre \& Omerzel (2015); } \\
\text { Martinez-Roman et al (2015); Pivcevic } \\
\text { \& Pranicevic, 2012); Jacob et al. (2003); } \\
\text { Ottenbacher e Gnoth (2005); Pikkemaat } \\
\text { \& Peters (2006); Lee t al. (2006), Tejada \& } \\
\text { Moreno (2013). }\end{array}$ \\
\hline
\end{tabular}

Fonte: Elaboração própria.

As hipóteses do estudo são:

H1: Os diretores de hotéis percebem a inovação como algo positivo e há uma preocupação em implementar inovações constantemente.

H2: Clientes, parceiros e funcionários contribuem para a implementação das inovações nos hotéis.

H3: Ao participar de eventos e associações, diretores adquirem conhecimento e estreitam relações importantes que também contribuem para implementação de inovações no hotel.

\section{ANÁLISE DE DADOS}

Para apreciar os dados, empregou-se a análise de conteúdo, que constitui um conjunto de técnicas de análise de comunicações, o qual tem como objetivo ultrapassar as incertezas e enriquecer a leitura dos dados coletados. Tal como assevera Chizzotti (2006, p. 98), "o objetivo da análise de conteúdo é compreender criticamente o sentido das comunicações, seu conteúdo manifesto ou latente, as significações explícitas ou ocultas".

No que se refere às diferentes etapas inerentes à análise de conteúdo, foram consideradas as orientações de Bardin (2006): 1) pré-análise, 2) exploração do material e 3) tratamento dos resultados, inferência e interpretação. Na primeira fase, organizou-se o material a ser analisado 
com o objetivo de torná-lo operacional, sistematizando as ideias iniciais. A segunda fase consistiu na exploração do material com a definição de categorias (sistemas de codificação) e a identificação das unidades de registro e das unidades de contexto nos documentos. Essa etapa é fundamental, pois pode permitir ou não a riqueza das interpretações e das inferências, a partir da descrição analítica e do material textual coletado submetido a um estudo aprofundado, orientado pelas hipóteses e pelos referenciais teóricos. Dessa forma, a codificação, a classificação e a categorização são básicas nesta fase. Para garantir o rigor científico, foi utilizado o software de análise de dados WebQDA. Na terceira etapa, ocorreu a condensação e o destaque das informações para análise, culminando nas interpretações inferenciais. Para Bardin (2006), esse é o momento da intuição, da análise reflexiva e crítica (Bardin, 2006).

\section{RESULTADOS}

\section{CARACTERIZAÇÃO DA AMOSTRA}

Foi feita a codificação por meio de "Descritores" do WebQDA. A amostra foi caracterizada quanto ao gênero ( 2 homens, 2 mulheres); grau de formação (2 Licenciados em Gestão Hoteleira e 2 em Administração de Empresas), tempo de atuação como diretor(a) (que variou entre 10 anos e 5 meses a 18 anos e 2 meses), idade (43,50, 65 e 83 anos), cargo (apenas 1 diretor entrevistado tem contrato de gestão por tempo indeterminado, os demais são os proprietários desde a fundação dos hotéis). Essas particularidades são condizentes com o previsto por Orfila-Sintes et al. (2005) e, por fim, as principais características relativas aos empreendimentos: dos 4 hotéis analisados, 3 são Pequenas Empresas, de 2 e 4 estrelas, com mais de 18 anos de existência no mercado e possuem pelo menos 6 funcionários. Apenas 1 é Média Empresa, com 70 anos, 3 estrelas e com 107 funcionários, trabalhando sob o regime de carteira assinada. Os entrevistados foram identificados pelos códigos EA, EI, EM e ES. 


\section{ANÁLISE E INTERPRETAÇÃO DOS RESULTADOS}

Outra codificação feita por meio do WebQDA, foi a por "Nós árvore". As categorias principais de análise, nomeadamente, concepção e importância (para o hotel) da inovação, criação de novos produtos e serviços e melhoria dos serviços existentes, formação de colaboradores e fontes externas de conhecimento para implementação de inovações, permitiram que subcategorias emergissem, de modo que as hipóteses do estudo foram respondidas.

Em relação à concepção de inovação, foram mencionados pelos entrevistados: "Qualquer coisa nova", "mais serviços de qualidade", "aumento da categoria hoteleira (por estrelas)", "ideias originais", "aquisição de novos equipamentos e recursos", "reformas infraestruturais", "adesão a ferramentas que contribuem para melhoria de serviços, mais eficiência, maior rapidez e qualidade", "forma como se gerem ferramentas inovadoras" e "tudo que é prático e permite à empresa estar na vanguarda." O entrevistado EA assevera: "Inovação? É qualquer coisa nova. (...). Se a gente muda e muda para melhor, isso também é inovação".

Embora o fragmento contemple o pensamento de Schumpeter (1934), é pertinente esclarecer que, sobretudo no campo empresarial, por trás da criação ou da implementação de algo está a garantia de um retorno financeiro. $O$ mesmo se aplica às melhorias significativas que também são consideradas pelo autor e pelos que nele se apoiam para definir inovação (por exemplo, Hjalager, 2010; Camisón \& Monfort Mir, 2012; OECD, 2005, entre outros)

EI acrescentou que a inovação "é todo tipo de ferramenta que possa contribuir para aumentar a eficiência, a qualidade e a rapidez do serviço (...). A inovação não é só ferramentas, e ideias é, portanto a forma como se gere determinadas ferramentas inovadoras."

Por sua vez, para EM a inovação pode ser: "aquisição de novos equipamentos e recursos", "reformas infraestruturais (...)". A expressão desses conceitos, além de contemplar e reforçar o sentido da inovação defendido pelos autores mencionados, evidencia as variações quanto aos tipos de inovações existentes, apontando a inovação como um desempenho ou resultado (Schumpeter,1934, 
Camisón \& Monfort- Mir, 2012, Hjalager, 2010 e Manual de Oslo - OECD, 2005). $O$ sentido da palavra "qualidade", mencionada por EI e EM, faz alusão ao atendimento às expectativas, às necessidades e aos padrões exigidos por seus clientes.

AentrevistadaES, numa perspectiva ainda alinhadaa dos demais entrevistados, associa o significado de inovação a uma particularidade hoteleira apresentada por Orfila-Sintes et al. (2005): a classificação por estrelas, que pode determinar ou condicionar o desempenho ou capacidade inovadora do hotel: "Inovação tem a ver com ideias originais, é poder oferecer mais serviços de qualidade (...). Quando um hotel teve aumento da categoria hoteleira, todo mundo sabe que isso aconteceu porque ele é inovador". De facto, quanto mais estrelas o hotel possui, maior é o número de serviços e produtos que este é obrigado a oferecer (dentro dos padrões de qualidade, exigido pelos órgãos de fiscalização competentes). No entanto, pode haver casos de hotéis que implementam inovações e serviços além do que é exigido em função da sua categoria. Afinal, essa é ainda uma questão para a qual não há consenso na literatura.

Quanto à importância da inovação, os diretores afirmaram que é: "uma vantagem competitiva", "permite o alcance de resultados financeiros", "busca incessante pela melhoria e diferencial de serviços", "tornar o hotel diferenciador "e "tornar o serviço mais rápido para o cliente". Essas concepções também se alinham com a literatura representada principalmente por Orfila-Sintes et al. (2005); Ulwick (2005); Orfila-sintes \& Mattsson (2009); Čivre \& Omerzel (2015); Martínez-Román et al. (2015); Saunila (2016), os quais enfatizam o poder da inovação de impactar positivamente o desempenho empresarial. Desempenho este que será tão ou mais favorável à medida em que os seus diretores tenham a correta noção do que a inovação é e dos benefícios que esta pode trazer.

Com efeito, as palavras de EI contemplam as falas dos outros entrevistados, "A inovação tem uma importância muito grande, essencialmente para o alcance de resultados financeiros para uma entidade e para se distinguir temporariamente, porque depois isto deixa de ser inovação e passa a ser comum às pessoas (...)". Tal fragmento revela a consciência da necessidade de tornar a inovação uma prática continuada. Os dados até então apresentados confirmam a hipótese de que os diretores hoteleiros percebem a inovação como algo positivo e preocupam-se em implementar inovações constantemente. 
A "Criação de novos produtos ou serviços elou melhorias de serviços existentes", sinteticamente entendida como a implementação de inovações, foi um dos quesitos analisados. E válido dizer que as respostas dos entrevistados foram agrupadas em 6 categorias e subcategorias, que são mostradas na Tabela 2.

Tabela 2: Implementação de inovações nos últimos três anos.

Criação de novos produtos ou serviços elou melhorias de serviços existentes

Categorias

Resultantes de necessidades obtidas junto aos clientes

Resultantes de sugestões de funcionários

Resultantes de parcerias

Pequenas mudanças e reparos infraestruturais

Mudanças por razões ambientais

Certificações
Ações implementadas (subcategorias)

Adoção de cartão-chave (magnético), de ar condicionado nos quartos, armazenamento e aquecimento das águas para o hotel e para os quartos com estruturas modernas, aquisição de leitor de cartão de identificação de estrangeiro, de cidadão e de passaporte, velocidade da internet; aquisição de novos canais de TV nos quartos e inserção de novos alimentos (inclusive antialérgicos) no cardápio do pequeno almoço, aquisição de software gestão integrada.

Oferta de novos alojamentos locais com preços mais econômicos, serviços de restauração, decoração, montagem dos diversos serviços. Disponibilidade de mesa com alimentos sem glúten no pequeno almoço, oferta de novos serviços (a incluir alimentos novos no cardápio de pequeno almoço).

Adoção de novos canais de venda (publicidade) e realização de eventos no hotel.

Atualização do wifi, de uma central telefônica, a introdução de chaves de cartão e consertos, atualização de site.

Redução do fluxo de água e da aquisição de equipamentos que consomem menos energia.

Obtenção de Certificação de qualidade pela LUSAENOR, HACCP, ISO 9001.

Fonte: Elaboração própria.

A leitura da Tabela 2 indica que clientes, parceiros e funcionários exercem influências e contribuem para a implementação das inovações nos hotéis, indicando que diretores têm perfil do tipo aberto e democrático. Isso induz ao entendimento de que a inovação é (ou tende a ser) iniciada a partir das interações pessoais, conforme foi previsto por Orfila-Sintes et al. (2005). Muitas dessas ações podem ser contempladas em mais de uma das cinco categorias de inovação propostas por Hjalager (2010), nomeadamente (produto, processo, gestão, marketing e inovações institucionais), dificultando a clara distinção e caracterização entre elas, tal como afirmou Gomezelj (2016).

As inovações implementadas em função da captação de necessidades junto aos clientes foram relativamente mais expressivas. Os diretores afirmaram que conheciam seu público-alvo e o identificaram no momento da entrevista, 
ISSN: 1983-7151

estratégia essa que é considerada por Ottenbacher e Gnoth (2005) como sendo bem-sucedida e favorável à implantação de mudanças ou melhorias significativas na empresa.

De acordo com o relato dos entrevistados, uma das formas de se perceber essas necessidades é por meio de questionários, por meio dos quais o cliente tem a oportunidade de avaliar serviços e sugerir novos ou melhorados produtos e serviços, tal como evidencia o relato de EM: “(...) Damos diariamente, uma bolsinha com um chocolatinho e um questionário com a abertura de cama.(...) Então, essa é a forma que temos para captar a atenção do cliente para nos deixar sugestões e eles deixam. Outra maneira comum é através do contato pessoal".

Todos os entrevistados afirmaram ter a preocupação de avaliar a satisfação de seus clientes. Unanimemente, foi revelado que contatam e questionam essa satisfação pessoalmente. Evidências como questionários de avaliação (digitais ou impressos) também foram apresentadas durante as entrevistas. Conforme relatou EA: "Não há cliente que saia daqui que não seja questionado se correu tudo bem, o que que o apetece que isto não tem e testamos essas sugestões, essas opiniões". ES complementa: "Isto é uma forma de mostrar para os clientes que eles são importantes, não é? (...) Uma grande parte das nossas inovações são para eles e é deles mesmo que elas vêm". A partir dos relatos, infere-se que tal estratégia de avaliação se traduz numa maneira bem-sucedida de captação de necessidades de mercado e melhoria do desempenho inovador do hotel. Além disso, o que pode ser introduzido como uma nova variante de serviço, desenhada para a satisfação de uma determinada necessidade dos clientes, pode também posteriormente ser entendido como o começo de uma modificação (radical) nas atividades do serviço que levam a tomar um rumo diferente ao idealizado inicialmente (Tether, 2005; Pikkemaat $\&$ Peters, 2006).

Fragmentos do relato de EA revelam como funcionários também podem influenciar a implementação de inovações: "Tenho aqui uma funcionária que deu me a ideia de oferecer aos hóspedes, pão quente todos os dias no pequeno almoço. (...) vimos a viabilidade disto (...) e então, passamos a produzir o pão nosso de cada dia para os clientes, eles adoram (...). Por aqui, não conheço nenhum hotel que faça isso" (...)." 
A qualificação de colaboradores foi mencionada como uma preocupação constante dos diretores, revelando a importância dos recursos humanos para o setor. Em todos os casos, foi confirmado pelos diretores que os seus colaboradores costumam estar envolvidos em todos os processos de criação e implementação de inovações nos hotéis. Tal resultado corresponde às constatações de Jacob et al. (2003), Pikkemaat \& Peters (2006), Orfila-Sintes et al. (2005); Orfila-sintes e Mattsson (2009); Hjalager (2010); Camisón \& MonfortMir, (2012); Pivcevic \& Pranicevic (2012). A realização de formações (no mínimo duas, por ano), que podem ocorrer internamente (envolvendo também a direção) ou externamente (com o incentivo a participação destes em cursos, eventos e ao compartilhamento de informações dentro das empresas), constitui evidências apresentadas pelos diretores.

Resultados permitiram confirmar também a hipótese 3: Ao participar de eventos e associações, diretores adquirem conhecimento e estreitam relações importantes que contribuem para aumentar o desempenho inovador. Ou seja, feiras, eventos, associações, cursos e similares foram reconhecidos como sendo uma fonte de conhecimento externa, que contribui para estimular a criatividade, resolver problemas e melhorar o relacionamento com clientes, parceiros, colaboradores (tal como dito por Martínez-Román et al., 2015). Nesse sentido, eventos como World Travel Marketing, a Feira de Turismo (FITUR), que ocorrem todos os anos, foram mencionados como exemplos de participação obrigatória pelos entrevistados.

A firmação de parcerias mostrou-se importante para estimular e fazer surgir novos canais de divulgação (inovações de marketing) dos hotéis. Neste caso, embora a publicidade tenha sido o resultado pretendido e real em todos os casos, também resultou em novos serviços (por exemplo: eventos à disposição dos clientes). Essas evidências parecem confirmar parcialmente a teoria de que os hotéis que pertençam a uma rede ou atuam em parcerias estreitam relações comerciais, aumentam possibilidades de vendas, reduzem riscos, custos e, assim, tendem a aumentar a sua capacidade de inovação (Tejada \& Moreno, 2013; Martínez-Román et al., 2015; Pikkemaat \& Peters, 2006). Até aqui, os dados confirmam a segunda hipótese: clientes, parceiros e funcionários exercem influências e contribuem para a implementação das inovações nos hotéis. 
Mudanças por razões ambientais, nomeadamente ações como redução do fluxo de água e aquisição de equipamentos que consomem menos energia, foram citadas como preocupações constantes, seja em virtude de pressões de clientes, autoridades ou para a obtenção de certificações e se traduzem como formas de racionalizar recursos naturais e essencialmente reduzir custos, além de melhorar a imagem do hotel perante seus clientes. Isso corrobora ideias de Le et al. (2006).

Pequenas mudanças e reparos infraestruturais foram citados muito frequentemente. O comentário de EA faz alusão a isso: "Apenas pequenas coisas, que não tem assim tanta expressão, não é? Por exemplo, uma atualização do wifi, de uma central telefônica, a introdução de chaves de cartão, são inovações, mas não é uma inovação assim de vulto". Isso foi previsto por Pikkemaat \& Peters (2006), que afirmaram que grande parte das inovações nos hotéis é pequena e superficial.

Verificou-se a partir das entrevistas que diretores também inovam por meio da introdução de $P \& D$, incorporada pela aquisição de novos equipamentos ou softwares, em vez de desenvolver atividades internas, como ocorre na indústria de produção de bens. Atualização de sites, aquisição de softwares e outras tecnologias junto a empresas especializadas evidenciam tal realidade. A incorporação dessas tecnologias pode ser traduzida em uma vantagem competitiva, seja pela eficiência produtiva (redução de custos), seja pela capacidade aprimorada de diferenciação (Hjalager 2002; Orfila-sintes \& Mattsson, 2009).

A obtenção de certificações de qualidade e outras como HACCP e ISO 9001 requer o cumprimento de uma série de exigências, que induz os hotéis a implementarem melhorias constantes. Apenas um hotel (4 estrelas) afirmou ter conquistado tais certificações. Isso foi decorrência da própria pressão e exigência do órgão fiscalizador competente em virtude da classificação que tal hotel apresenta.

CONCLUSÕES

Cumprindo o objetivo proposto, este trabalho mostrou a percepção de diretores hoteleiros em Aveiro no tocante à inovação, que unanimemente foi 
relatada como muito relevante, constituindo assim uma preocupação constante, em virtude das vantagens oferecidas, que segundo os entrevistados vão desde a redução de custos ao aumento de competitividade.

Assim, de modo coerente, na visão dos diretores, o conceito de inovação referindo-se a resultado ou ao desempenho propriamente dito foi traduzido em conformidade com definições-chave na literatura, tal como mostra alguns exemplos: "coisa nova", "aquisição de novos equipamentos e recursos", "reformas infraestruturais", "adesão a ferramentas que contribuem para melhoria de serviços", "mais eficiência e qualidade", "forma como essas ferramentas são geridas"

A pesquisa revelou que clientes, parceiros e funcionários exercem influências e contribuem para a implementação das inovações nos hotéis, revelando um perfil aberto e democrático dos diretores. Tal resultado também levou à constatação de que as interações pessoais constituem o ponto de partida para o processo de criação, implementação e mesmo de avaliação de ações inovadoras (majoritariamente resultantes do contato direto com clientes) em tais empreendimentos, que podem estar agrupadas em cinco tipos (produto, processo, gestão, marketing e institucional) muito relacionados entre si.

A qualificação de colaboradores foi mencionada como uma preocupação constante dos diretores, revelando a importância dos recursos humanos para o setor. A realização de formações (no mínimo, duas por ano), que podem ocorrer internamente (envolvendo também a direção) ou externamente (com o incentivo à participação destes em cursos, eventos e ao compartilhamento de informações dentro das empresas), constituem evidências apresentadas pelos diretores.

Mencionadas muito frequentemente em todos os casos, ações que se classificam como pequenas mudanças e reparos infraestruturais permitiram a constatação de que as inovações nos hotéis são predominantemente do tipo incrementais e superficiais. Nesse sentido, grande parte delas se traduzem em formas de racionalizar recursos naturais e essencialmente reduzir custos, além de melhorar a imagem do hotel perante seus clientes.

Assim como a obtenção de certificações não constitui um indicador principal da inovação em hotéis, diferente do que ocorre no setor industrial, outra particularidade diz respeito às atividades de $P \& D$. Tais atividades são 
incorporadas pela aquisição de novos equipamentos ou softwares, em vez de serem desenvolvidas internamente. $O$ relato de mudanças como a atualização de sites, aquisição de softwares e outras tecnologias junto a empresas especializadas confirmam tal realidade no contexto investigado.

Embora o número muito reduzido de entrevistados seja apontado com a principal limitação deste estudo, foi possível confirmar hipóteses e aspectos do setor hoteleiro à luz da literatura da inovação no turismo e apreender variáveis importantes a serem consideradas no processo de medição da inovação. Essa constitui uma necessidade para um tão complexo e abrangente setor, como o turismo, que apresenta particularidades muitas vezes desconsideradas por instrumentos de medição do desempenho inovador desenvolvidos para a indústria manufatureira.

Com vistas a contribuir para este fim, recomenda-se o desenvolvimento de pesquisas quali e quantitativas, as quais, valendo-se de inquéritos (contendo questões abertas e fechadas), busquem ampliar o alcance de resultados apresentados. As questões aqui propostas poderiam ser aplicadas nos mais distintos meios de hospedagem e/ou outros ramos do turismo em diferentes contextos, de modo a permitir que comparações sejam feitas e contribuam para tornar mais sólido o conhecimento e o campo de investigação científica do setor.

\section{REFERÊNCIAS}

Alvarez Gil M. J.; Burgos, Jiménez J; Céspedes, Lorente J. J. (2001). An analysis of environmental management, organizational context and performance of Spanish hotels. Omega; 29:457-71.

Bardin, L. (2006). Análise de conteúdo (L. de A. Rego \& A. Pinheiro, Trads.). Lisboa: Edições 70. (Obra original publicada em 1977)

Câmara de Aveiro. (2017). Turismo com crescimento continuado em Aveiro. Disponível em: https://www.noticiasaominuto.com/economia/732350/turismo-com-crescimentocontinuado-em-aveiro. Consultado em 07. Jul. 2017.

Camisón C. (2000). Strategic attitudes and information technologies in the hospitality business: an empirical analysis. Hospitality Management;19:125-43.Bardin, L. (2006). Análise de conteúdo (L. de A. Rego \& A. Pinheiro, Trads.). Lisboa: Edições 70. 
Camisón, C., \& Monfort-Mir, V. M. (2012). Measuring innovation in tourism from the Schumpeterian and the dynamic-capabilities perspectives. Tourism Management, 33(4), 776-789. http://doi.org/10.1016/j.tourman.2011.08.012

Chizzotti, A. (2006). Pesquisa em ciências humanas e sociais (8a ed.). São Paulo: Cortez.

Choi, B; Poon, S.K; Davis, J.G. (2008). Effects of knowledge management strategy on organizational performance: a complementarity theory-based approach. Omega; 36, in press.

Čivre, Ž., \& Omerzel, D. G. (2015). The behaviour of tourism firms in the area of innovativeness. Economic Research- Ekonomska Istrazivanja, 28(1), 312-330. http://doi.org/10.1080/13316 $77 X .2015 .1043778$

Creswell, J. W. (1998). Qualitative inquiry and research design: choosing among five traditions. Thousand Oaks, California: Sage Publications.

Dencker, A. de F. M. (1998). Métodos e técnicas de pesquisa em turismo. São Paulo: Editora Futura.

Dwyer, L., Forsyth, P., \& Dwyer, W., (2010). Tourism economics and policy. (2a ed.). Bristol: Channel View Publications.

Gomezelj, Doris Omerzel. (2016),"A systematic review of research on innovation in hospitality and tourism", International Journal of Contemporary Hospitality Management, 28(3), $516-558$.

Hall, C. M., \& Williams, A. M. (2008). Tourism and innovation. New York: Routledge. Haugland,

Harrington, R. J. (2004). The environment, involvement, and performance: implications for the strategic process of food service firms. Hospitality Management, 23:317-41.

Hjalager A. M. (2002). Repairing innovation defectiveness in tourism. Tourism Management, 23(5), 465-74.

Hjalager, A. M. (2010). A review of innovation research in tourism. Tourism Management, 31(1), 1-12. http://doi.org/10.1016/j.tourman.2009.08.012

Instituto Nacional de Estatística [INE] (2010). Anuário Estatístico da Região Centro. Lisboa: Instituto Nacional de Estatística.

Jacob, M., Tintoré, J., Aguiló, E., Bravo, A., \& Mulet, J. (2003). Innovation in the tourism sector: Results from a pilot study in the Balearic Islands. Tourism Economics, 9(3), 279-295. Retrieved from http://www.scopus.com/inward/ 
Krizaj, D., Brodnik, A., \& Bukovec, B. (2014) A Tool For Measurement of Innovation Newness and Adoption in Tourism Firms. International Journal of Tourism Research, 16: 113-125. Disponível em: http://doi.org/10.1002/jtr

Le, Y; Hollenhorst, S; Harris, C; Mclaughlin, W; Shook, S. (2006). Environmental management - a study of vietnamese hotels. Annals of Tourism Research, v. 33, n. 2, pp. 545-567.

Martínez-román, J. A., Tamayo, J. A., Gamero, J., \& Romero, J. E. (2015). Innovativeness and business performances in tourism SMEs. Annals of Tourism Research, 54, 118-135. http:// doi.org/10.1016/j.annals.2015.07.004

Marujo, A. (2013). A pesquisa em turismo: reflexões sobre as abordagens qualitativa e quantitativa. TURyDES, Revista de investigación en Turismo y Desarrollo Local, 6 (14), 1-16. Recuperado de: http://www.eumed.net/rev/turydes/14

Minayo, Maria Cecília de Souza (org.). (2010). Pesquisa social: teoria, método e criatividade. 29. ed. Petrópolis, RJ: Vozes

Miles, M.B.; Huberman, A. M. (1994). Qualitative data analysis: an expanded sourcebook California: Sage.

OCDE. Manual de Oslo. (2005). $3^{\mathrm{a}}$ ed. FINEP/OECD.

Ottenbacher M, Gnoth J. (2005). How to develop successful hospitality innovation. Cornell Hotel and Restaurant Administration Quarterly, 46(2):205-22.

Orfila-Sintes, F., Crespí-Cladera, R., \& Martínez-Ros, E. (2005). Innovation activity in the hotel industry: Evidence from Balearic Islands. Tourism Management, 26(6), 851-865. http://doi. org/10.1016/j.tourman.2004.05.005

Orfila-sintes, F., \& Mattsson, J. (2009). Innovation Behavior in the Hotel Industry. Omega - The International Journal of Management Science, (37), 380 - 394. doi.org/10.1016/j. omega.2007.04.002

Patton, M. G. (2002). Qualitative Research and Evaluation Methods. Thousand Oaks, CA: Sage.

Pikkemaat, B., \& Peters, M. (2006). Towards the Measurement of innovation - a pilot study in the Small and Medium Sized Hotel industry. ... of Quality Assurance in Hospitality \& Tourism, 6, 89-112. http://doi.org/10.1300/J162v06n03

Pivcevic, S., \& Pranicevic, D. G. (2012). Innovation activity in the hotel sector - The case of Croatia. Ekonomska Istrazivanja, 1(SPEC. ISS. 1), 337-363. Retrieved from http://www. scopus.com/inward 
Saunila, M. (2016). Performance measurement approach for innovation capability in SMEs. International Journal of Productivity and Performance Management, 65(2), 162-176. http:// doi.org/10.1108/IJPPM-08-2014-0123

Schumpeter, J. (1934). Teoria do desenvolvimento econômico. São Paulo: Abril Cultural.

Sharma, D. (2016). Enhancing customer experience using technological innovations: A study of the Indian hotel industry. Tourism Themes, 8(4), 469-480. https://doi.org/10.1108/ WHATT-04-2016-0018

Silva, J. A., Matias, A., \& Pintassilgo, P. (2014). Reflexões sobre a economia do turismo. In: C. Costa; F. Brandão; R. Costa \& Z. Breda (eds.). Produtos e competitividade do turismo na Lusofonia. (p.41-57). Aveiro: Escolar Editora.

Tejada, P., \& Moreno, P. (2013). Patterns of innovation in tourism "Small and Medium-size Enterprises." The Service Industries Journal, 33(7-8), 749-758. http://doi.org/10.1080/0264 2069.2013.740469

Tether, B. S. (2005). Do services innovate (differently)? Insights from the European innobarometer survey. Industry \& Innovation, 12(2), 153-184.

Thomas, R., \& Wood, E. (2014). Innovation in tourism: Re-conceptualising and measuring the absorptive capacity of the hotel sector. Tourism Management, 45, 39-48. http://doi. org/10.1016/j.tourman.2014.03.012

Van der Aa, W.; Elfring, T. (2002). Realizing innovation in services. Scandinavian Journal of Management, 18: 155-171.

Vieira, M. M. F. (2004). Por uma boa pesquisa (qualitativa) em administração. In: Vieira, M. M. F.; Zouain, D. M. Pesquisa qualitativa em administração. Rio de Janeiro: editora FGV, pp.13-28.

Wright CM, Mechling G. (2002). The importance of operations management problems in service organizations. Omega 2002;30:77-87.

\section{CONTRIBUIÇÃO DOS AUTORES NA CONSTRUÇÃO DO ARTIGO}

AIRES: Elaboração e correção em conformidade com as exigências da Revista. 